Article

\title{
Investigation of Eigenmode-Based Coupled Oscillator Solver Applied to Ising Spin Problems
}

\author{
Shintaro Murakami, Okuto Ikeda, Yusuke Hirukawa and Toshiharu Saiki * \\ Graduate School of Science and Technology, Keio University, Yokohama 223-8522, Japan; \\ murashin1218@keio.jp (S.M.); okuto.ikeda@saiki.elec.keio.ac.jp (O.I.); \\ yusuke.hirukawa@saiki.elec.keio.ac.jp (Y.H.) \\ * Correspondence: saiki@elec.keio.ac.jp
}

check for updates

Citation: Murakami, S.; Ikeda, O.; Hirukawa, Y.; Saiki, T. Investigation of Eigenmode-Based Coupled Oscillator Solver Applied to Ising Spin Problems. Symmetry 2021, 13, 1745. https://doi.org/10.3390/ sym13091745

Academic Editor: David Waldeck

Received: 6 August 2021

Accepted: 15 September 2021

Published: 19 September 2021

Publisher's Note: MDPI stays neutral with regard to jurisdictional claims in published maps and institutional affiliations.

Copyright: (c) 2021 by the authors. Licensee MDPI, Basel, Switzerland. This article is an open access article distributed under the terms and conditions of the Creative Commons Attribution (CC BY) license (https:// creativecommons.org/licenses/by/ $4.0 /)$.

\begin{abstract}
We evaluate a coupled oscillator solver by applying it to square lattice $(\mathrm{N} \times \mathrm{N})$ Ising spin problems for $\mathrm{N}$ values up to 50 . The Ising problems are converted to a classical coupled oscillator model that includes both positive (ferromagnetic-like) and negative (antiferromagnetic-like) coupling between neighboring oscillators (i.e., they are reduced to eigenmode problems). A map of the oscillation amplitudes of lower-frequency eigenmodes enables us to visualize oscillator clusters with a low frustration density (unfrustrated clusters). We found that frustration tends to localize at the boundary between unfrustrated clusters due to the symmetric and asymmetric nature of the eigenmodes. This allows us to reduce frustration simply by flipping the sign of the amplitude of oscillators around which frustrated couplings are highly localized. For problems with $\mathrm{N}=20$ to 50, the best solutions with an accuracy of $96 \%$ (with respect to the exact ground state) can be obtained by simply checking the lowest $\sim \mathrm{N} / 2$ candidate eigenmodes.
\end{abstract}

Keywords: combinatorial optimization; Ising spin glass; coupled oscillator; eigenmode; clustering

\section{Introduction}

The spin glass model originates from condensed matter physics, where it was applied to physical systems in which magnetic atoms are randomly distributed in a non-magnetic host and induce ferromagnetic and antiferromagnetic interactions between neighboring magnetic moments. The physical system is mathematically modeled by a weighted graph where each vertex corresponds to a spin and each edge represents the interaction between spins with positive and negative signs [1,2]. In the Ising spin model, the spin is a binary variable that takes the value $\pm 1[3,4]$. The Ising problem is to find a binary spin configuration that minimizes the total energy function (the number of frustrated edges) for a given set of edges. A variety of combinatorial optimization problems, such as sequencing and ordering problems, resource allocation problems, and clustering problems $[5,6]$, can be mapped to the Ising problem [7].

To solve the Ising problem using a brute force combination approach, we need to check $2^{n}$ possibilities, where $n$ is the total number of spins. The branch and bound method, which based on a tree search algorithm, is commonly used to find the exact ground state without an exhaustive search [8]. However, this method still requires a lot of CPU time and memory and is only applicable to instances with a small number of spins. The potential applications of the Ising model to optimization problems have motivated the development of heuristic algorithms for finding high-quality solutions for instances with a large number of spins [9,10]. Although heuristic algorithms generally do not guarantee an optimal solution, they can yield good time-to-solution in practice.

Simulated annealing (SA), one of the most common heuristic algorithms, mimics the physical process of annealing, where a material is slowly cooled to obtain the lowest energy state [11-13]. Its algorithm is based on Monte Carlo simulation. Starting with an initial spin configuration, a new candidate configuration is selected in each iteration 
of the simulation. If the total energy decreases for the new candidate, that candidate is accepted and the iterative process continues. Otherwise, it is accepted with a probability given by the Boltzmann factor, which decreases with temperature. This random acceptance allows the algorithm to escape local minima. The system eventually cools into the global minimum in the spin configuration space.

The growth of data size in Ising problems has spurred interest in physical hardware systems that directly minimize the energy function [14-21]. Such systems are called Ising machines. An example of an Ising machine is the quantum annealing machine from D-Wave Systems, which was implemented using superconducting qubits [22,23]. The machine operates at a cryogenic temperature. The connectivity between qubits is limited to rather simple structures. Quantum adiabatic optimization inspired a new heuristic algorithm for the Ising problem, called simulated bifurcation, which simulates adiabatic evolution of classical nonlinear oscillators that exhibit bifurcation [24,25].

We recently developed a heuristic algorithm for the Ising problem in which the Ising spin system is replaced by a coupled oscillator system, which is possible owing to the equivalence of their equations of motion [26]. We obtained exact ground states for problems with a small number of spins by simply calculating the lowest mode of the coupled oscillators (i.e., the lowest eigenvalue and eigenvector of the matrix representing the equations of motion). We also developed an error correction algorithm that modifies the coupling strength depending on the amplitude of individual oscillators. This heuristic algorithm is a kind of annealing process since the energy landscape in the dipole configuration space is optimized in such a way that the correct configuration is equivalent to the lowest eigenmode. Based on this concept, we proposed an Ising machine composed of plasmon particles with dipole-dipole interaction, whose strength can be modified by a phase-change material inserted between neighboring particles [27].

In the present paper, we reconsider the coupled oscillator solver (COS) described above from the following viewpoints: (1) the lowest mode of the coupled oscillators may not always provide a minimally frustrated spin configuration (exact ground state) for largesized Ising problems; and (2) it is desirable to replace the time-consuming error correction algorithm with a better algorithm inspired by an analysis of good candidate solutions (i.e., the lowest eigenmodes). We apply the COS to two-dimensional $\mathrm{N} \times \mathrm{N}$ oscillators for $\mathrm{N}$ values of up to 50. A map of the oscillation amplitudes (eigenvector components) of lower-frequency eigenmodes enables us to visualize unfrustrated clusters. We found that frustration tends to localize at the boundary between clusters due to the symmetric and asymmetric nature of the eigenmodes. This allows us to reduce frustration simply by flipping the sign of the amplitude of highly frustrated oscillators.

\section{Coupled Oscillator Solver Applied to Ising Spin Problems}

Here, we consider a square lattice $(\mathrm{N} \times \mathrm{N})$ Ising spin glass problem without an external magnetic field. The spin configuration that minimizes the Ising energy is given by

$$
E_{\text {Ising }}=-\sum_{i=1}^{N \times N} \sum_{j=1}^{N \times N} J_{i j} s_{i} s_{j}
$$

where $s_{i}$ denotes the $i$ th spin with a value of 1 or -1 , and $J_{i j}$ is the coupling coefficient between the $i$ th and $j$ th spins having both positive $(+J$; ferromagnetic coupling) and negative ( $-J$; antiferromagnetic coupling) values. In this study, only four nearest neighbor couplings are taken into account. For a given spin configuration, when $J_{i j} s_{i} s_{j}>0$, the coupling $J_{i j}$ is satisfied, otherwise it is frustrated. Minimizing the Ising energy is equivalent to maximizing the number of satisfied couplings.

We start with an instance of $10 \times 10(\mathrm{~N}=10)$ spins with 200 couplings. Problems were generated by randomly assigning ferromagnetic and antiferromagnetic couplings with a number ratio of 1:1. Figure 1a shows the distribution of frustrated couplings (bold lines) for the exact ground state provided by a public domain [28], where an algorithm in [29] is 
used. In this algorithm, the original graph underlying the Ising problem is transformed into a dual graph, and minimum weight perfect matching is calculated. For the backward transformation, the matching leads to an Eulerian subgraph, the weight of which is also minimized. Owing to the one-to-one correspondence between the Eulerian subgraphs (cycles) and cuts in the original graph, the optimum Eulerian subgraphs provide the exact ground state for the Ising problem. The red circles and blue diamonds represent up-spin and down-spin states, respectively. The number of satisfied couplings of the exact ground state was found to be $n^{\mathrm{E}}=166$.

(a)

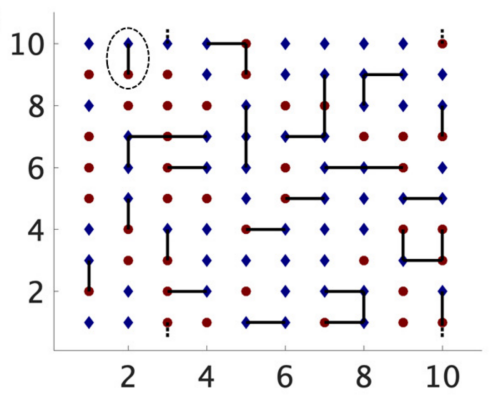

(c)

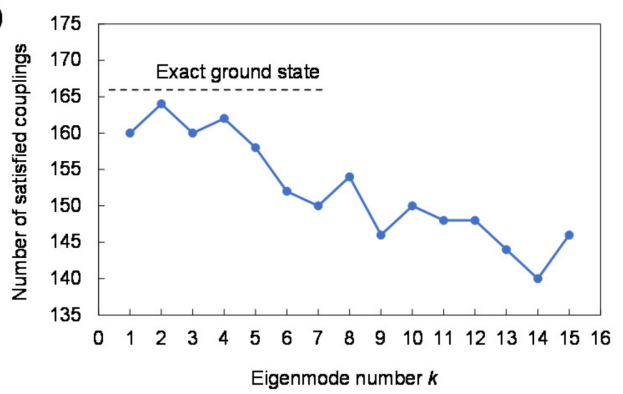

(b) $k=1$

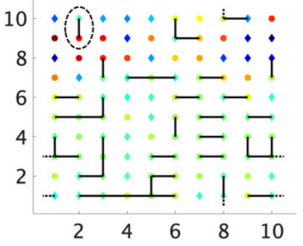

(d)

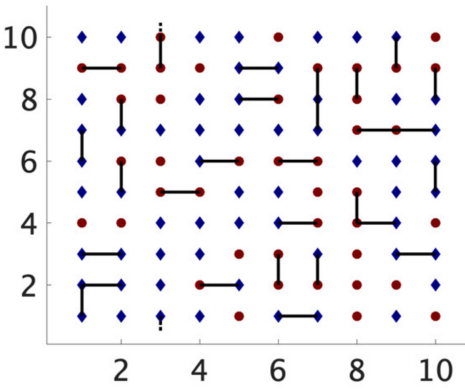

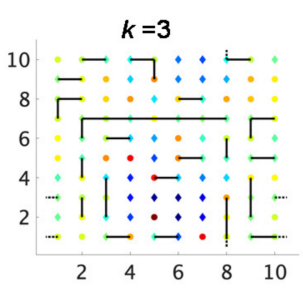

$k=4$

(f)

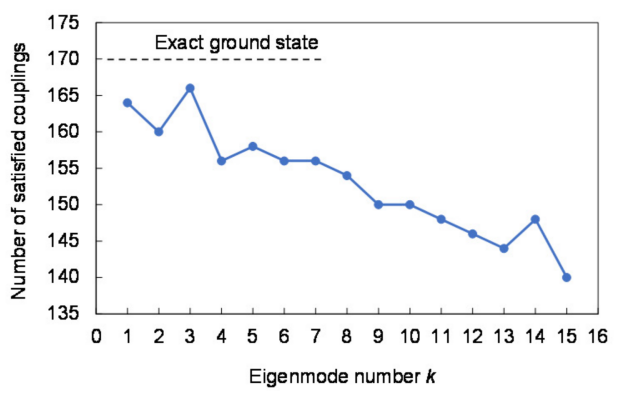

(e) $k=1$

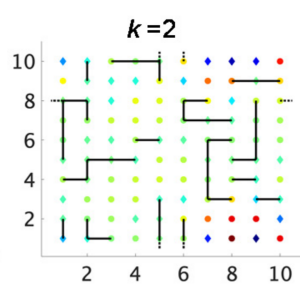

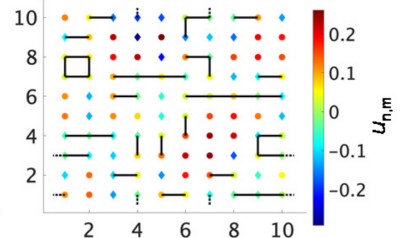

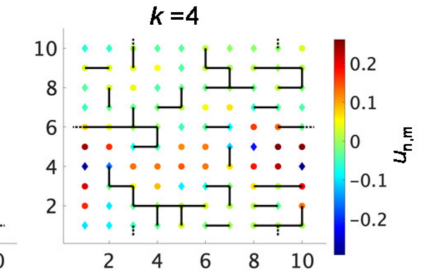

Figure 1. Instance of square lattice $(10 \times 10)$ Ising spin problem solved by the COS. (a) Spin configuration of the exact ground state. The red circles and blue diamonds represent up-spin and down-spin states, respectively. Frustrated couplings are indicated by bold lines. (b) Mapping of oscillation amplitude $u_{\mathrm{n}, \mathrm{m}}$ for the lowest four eigenmodes $k=1$ to 4 . The circles and diamonds respectively represent positive and negative signs of $u_{\mathrm{n}, \mathrm{m}}$. Frustrated couplings are indicated by bold lines. (c) Plot of the number of satisfied couplings $n^{\mathrm{CO}}$ as a function of eigenmode number $k$. (d-f) Results for another instance.

To solve the problem, we converted it to a classical coupled oscillator model by replacing the ferromagnetic and antiferromagnetic couplings with positive and negative couplings, respectively, between neighboring oscillators, as illustrated in Figure 2a. A normal attractive spring connecting neighboring masses gives rise to a positive interac- 
tion, and the masses tend to move in the same direction at lower frequency. A negative interaction is implemented by a repulsive spring (which does not exist in reality) to allow the masses to move in opposite directions. Due to the mathematical similarity between the inter-spin and inter-oscillator interactions, we anticipate that the sign of the oscillator amplitude for the lowest mode is the same or close to the spin configuration for the exact ground state for the original Ising problem.

(a)

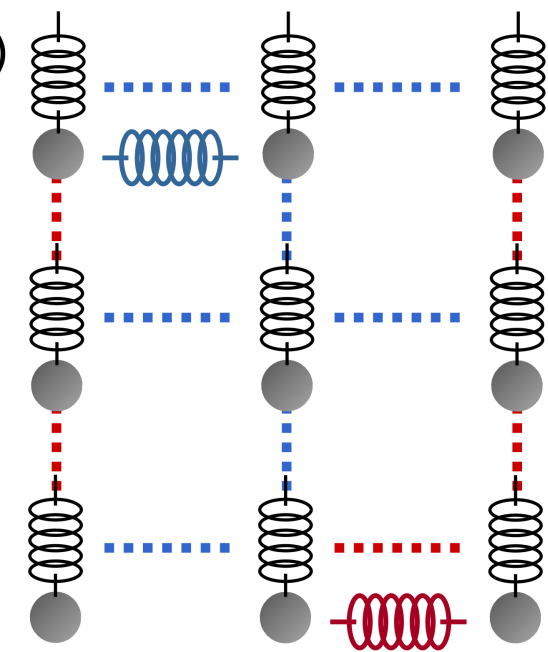

(b)

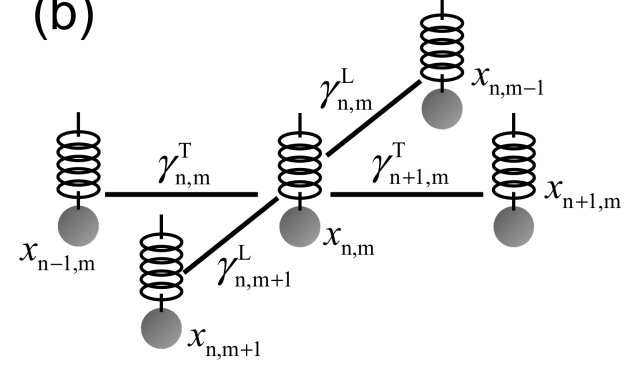

[...... : positive coupling

. . . . : : negative coupling

Figure 2. (a) Coupled oscillator model that includes both positive (ferromagnetic-like) and negative (antiferromagnetic-like) coupling. (b) Definition of the superscripts and subscripts associated with $\gamma$.

The equation of motion for the masses on the square lattice is given by

$$
\ddot{x}_{\mathrm{n}, \mathrm{m}}=\alpha^{2}\left(\gamma_{\mathrm{n}, \mathrm{m}}^{\mathrm{T}} x_{\mathrm{n}-1, \mathrm{~m}}+\gamma_{\mathrm{n}, \mathrm{m}}^{\mathrm{L}} x_{\mathrm{n}, \mathrm{m}-1}+\gamma_{\mathrm{n}+1, \mathrm{~m}}^{\mathrm{T}} x_{\mathrm{n}+1, \mathrm{~m}}+\gamma_{\mathrm{n}, \mathrm{m}+1}^{\mathrm{L}} x_{\mathrm{n}, \mathrm{m}+1}\right)
$$

where $x_{n, m}$ is the displacement of a mass at the (n, m) lattice site and $\alpha$ is the square root of the spring constant divided by the mass, which represents the strength of the coupling between neighboring oscillators. Here, $\gamma_{\mathrm{n}, \mathrm{m}}$ is +1 for positive interaction and -1 for negative interaction, and " $\mathrm{T}$ " and " $\mathrm{L}$ " indicate the transverse and longitudinal directions, respectively. The definition of the superscripts and subscripts associated with $\gamma$ is given in Figure 2b. The eigenmodes of the collective motion of oscillators can be calculated by substituting $x_{n, m}=u_{n, m} \exp (-\mathrm{i} \omega t)$ into Equation (2) to obtain

$$
\omega^{2} u_{\mathrm{n}, \mathrm{m}}=\alpha^{2}\left(\gamma_{\mathrm{n}, \mathrm{m}}^{\mathrm{T}} u_{\mathrm{n}-1, \mathrm{~m}}+\gamma_{\mathrm{n}, \mathrm{m}}^{\mathrm{L}} u_{\mathrm{n}, \mathrm{m}-1}+\gamma_{\mathrm{n}+1, \mathrm{~m}}^{\mathrm{T}} u_{\mathrm{n}+1, \mathrm{~m}}+\gamma_{\mathrm{n}, \mathrm{m}+1}^{\mathrm{L}} u_{\mathrm{n}, \mathrm{m}+1}\right)
$$

By assuming a periodic boundary condition for $\gamma_{n, m}$, Equation (3) for the $\mathrm{N} \times \mathrm{N}$ system can be reduced to the problem of calculating the eigenvalue (frequency) $\omega$ and eigenvector (amplitude) $u_{\mathrm{n}, \mathrm{m}}$ for $\mathrm{N}^{2}$ elements. Figure $1 \mathrm{~b}$ shows a map of the oscillation amplitude $u_{\mathrm{n}, \mathrm{m}}$ of the lowest four eigenmodes (eigenmode number $k=1$ to 4 ) for the original Ising problem. The circles and diamonds respectively represent positive and negative signs of $u_{\mathrm{n}, \mathrm{m}}$. The distribution of $u_{\mathrm{n}, \mathrm{m}}$ is not localized; it covers the entire system. If two neighboring oscillators connected by positive (negative) coupling move in opposite directions (the same direction), they are considered to be frustrated, analogous to the Ising spin model. Frustrated couplings are indicated by bold lines in Figure 1b. For instance, the two oscillators enclosed by the dotted ellipse in Figure $1 \mathrm{~b}$ move in opposite directions, as indicated by $u_{\mathrm{n}, \mathrm{m}}<0$ for the upper oscillator and $u_{\mathrm{n}, \mathrm{m}}>0$ for the lower oscillator. Since the coupling is positive, which is evident by the frustrated coupling (connected by the bold line) of the two corresponding spins with opposite signs shown in Figure 1a, the oscillators are also frustrated for the eigenmode with $k=1$. A lower frequency (eigenmode number) typically yields a smaller number of frustrated couplings (but not strictly, as demonstrated in Figure 1c). 
The number of satisfied couplings $n^{\mathrm{CO}}$ as a function of eigenmode number $k$ is plotted in Figure 1c. $n^{\mathrm{CO}}$ is maximized at the second lowest mode $(k=2)$ and tends to decrease with $k$. The maximum $n^{\mathrm{CO}}\left({ }^{\mathrm{CO}}\right.$ max $)$ is 164 , which corresponds to $98.7 \%$ of $n^{\mathrm{E}}(=166)$. Figure $1 \mathrm{~d}-\mathrm{f}$ show the results for another instance of the system. Also in this case, the distribution of the amplitude $u_{n, m}$ is delocalized. $n^{\mathrm{CO}}$ is a maximum at $k=3$, for which $n^{\mathrm{CO}}{ }_{\max }$ is 166 or $97.6 \%$ of $n^{\mathrm{E}}(=170)$. Table 1 summarizes the values of $k$ that maximize $n^{\mathrm{CO}}$ and $n^{\mathrm{CO}}{ }_{\max } / n^{\mathrm{E}}$ for nine different instances. For all cases, $n^{\mathrm{CO}}$ is a maximum at $k \leq 3$ and $n^{\mathrm{CO}} \max / n^{\mathrm{E}}$ is larger than $94.1 \%$.

Table 1. Values of $k$ required to maximize $n^{\mathrm{CO}}$ and $n^{\mathrm{CO}}$ max $/ n^{\mathrm{E}}$ for nine instances of problem with $\mathrm{N}=10$.

\begin{tabular}{ccccccccccc}
\hline Sample\# & 1 & 2 & 3 & 4 & 5 & 6 & 7 & 8 & 9 \\
\hline$k$ & 1 & 2 & 2 & 1 & 1 & 2 & 1 & 1 \\
\hline$n^{\mathrm{CO}}{ }_{\max } / n^{\mathrm{E}}(\mathbf{\%})$ & 94.2 & 95.3 & 98.8 & 96.4 & 96.6 & 96.5 & 96.4 & 98.8 & 97.6 \\
\hline
\end{tabular}

\section{Eigenmode Mapping to Visualize Frustration Localization}

Next, we increase the problem size to $20 \times 20(\mathrm{~N}=20)$. The distribution of frustrated couplings for the exact ground state is shown in Figure 3a. The problem was converted to the coupled oscillator model and the eigenvalues and eigenvectors were calculated. Figure $3 \mathrm{~b}$ shows a map of the oscillation amplitude and frustrated couplings for eigenmode numbers of $k=1$ to 5 . Figure 3d,e show the results for another instance. In contrast to the smaller problem with $\mathrm{N}=10$, the collective oscillation is spatially localized and clusters form depending on $k$. It is reasonable that the distribution of the signs of $u_{\mathrm{n}, \mathrm{m}}$ in each cluster is in complete agreement with that of the spin configuration in the exact ground state. The formation of such unfrustrated clusters can specify the region where frustration occurs with high probability since the oscillator system lowers the eigenfrequency by reducing the amplitude of the oscillators around which frustrated couplings are localized. In particular, for lower eigenmodes, the unfrustrated clusters tend to extend as widely as possible, making frustrated couplings as localized as possible at the boundary between unfrustrated clusters. In Figure 3b, there are many oscillators around which three of the four couplings are frustrated. For these oscillators, the number of frustrated couplings can be reduced from three to one by flipping the sign of the amplitude. Figure $3 \mathrm{c}$ shows $n^{\mathrm{CO}}$ before and after the flipping process as a function of $k$, demonstrating the effectiveness of flipping in reducing frustration. $n^{\mathrm{CO}}$ max is obtained for $k=4$ after flipping and $n^{\mathrm{CO}}{ }_{\max } / n^{\mathrm{E}}$ reaches $97.7 \%$. For the other problem (Figure $3 \mathrm{f}), n^{\mathrm{CO}} \max / n^{\mathrm{E}}$ is a maximum $(97.6 \%)$ at $k=3$.

Table 2 shows the results for nine instances, including the value of $k$ required to maximize $n^{\mathrm{CO}}$ and $n^{\mathrm{CO}}$ max $/ n^{\mathrm{E}}$ before and after flipping, to evaluate the effectiveness of flipping. For all cases, $n^{\mathrm{CO}}$ is a maximum at $k \leq 8$ and $n^{\mathrm{CO}}{ }_{\max } / n^{\mathrm{E}}$ after flipping is larger than $96.7 \%$. The eigenmode calculation is useful for visualizing unfrustrated clusters to find a fairly good solution, in which frustrated couplings are strongly localized around specific oscillators. The solution is effectively improved by flipping the sign of the amplitude to reduce frustration. It should be mentioned that it is relatively less probable for the lowest eigenmode $(k=1)$ to provide the highest $n^{\mathrm{CO}} \max / n^{\mathrm{E}}$. This might be due to the fact that the amplitude distribution has more nodes between unfrustrated clusters for a few higher eigenmodes and the frustration is more localized in the vicinity of the nodes. To summarize, what the COS does before flipping is to find the eigenmode consisting of clusters without nodes (locally symmetric), and simultaneously localizing nodes at the cluster boundaries (globally asymmetric). 
(a)

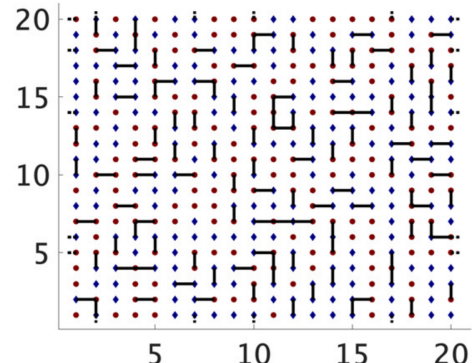

(c)

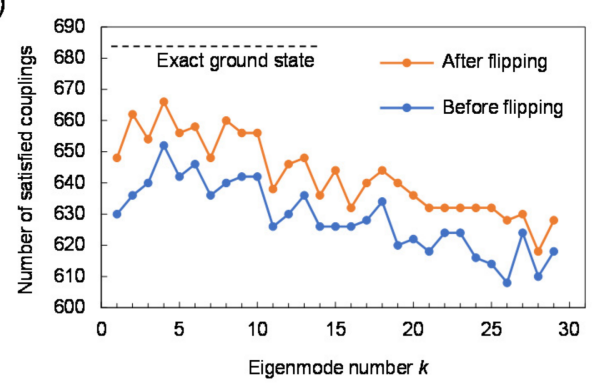

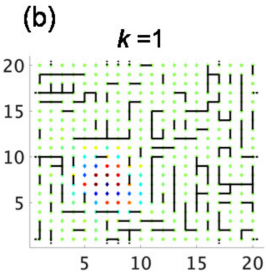

(d)

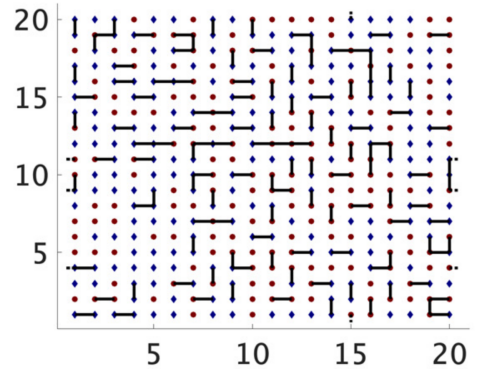

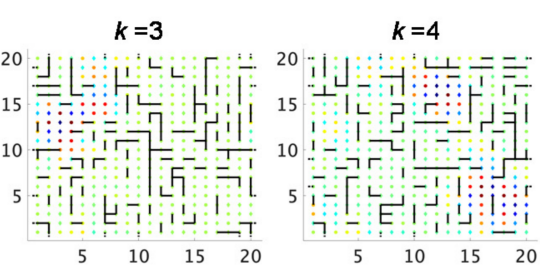

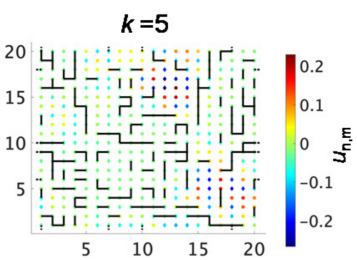

(f)
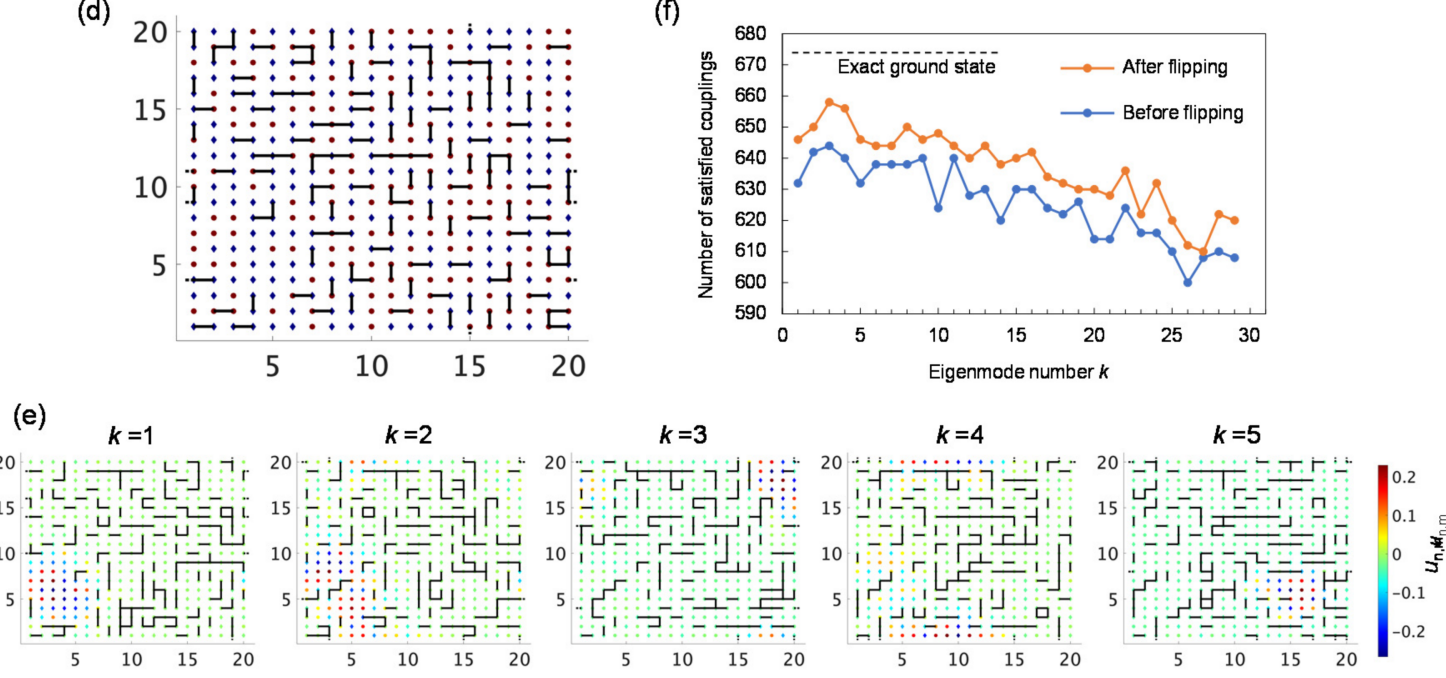

Figure 3. Instance of square lattice $(20 \times 20)$ Ising spin problem solved by the COS. (a) Spin configuration of the exact ground state. The red circles and blue diamonds represent up-spin and down-spin states, respectively. Frustrated couplings are indicated by bold lines. (b) Mapping of oscillation amplitude $u_{\mathrm{n}, \mathrm{m}}$ for the lowest five eigenmodes $k=1$ to 5 . The circles and diamonds respectively represent positive and negative signs of $u_{\mathrm{n}, \mathrm{m}}$. Frustrated couplings are indicated by bold lines. (c) Plot of the number of satisfied couplings $n^{\mathrm{CO}}$ before and after flipping as a function of eigenmode number $k$. (d-f) Results for another instance.

Table 2. Values of $k$ required to maximize $n^{\mathrm{CO}}$ and $n^{\mathrm{CO}}$ max $/ n^{\mathrm{E}}$ before and after flipping for nine instances of problem with $\mathrm{N}=20$.

\begin{tabular}{cccccccccc}
\hline Sample\# & 1 & 2 & 3 & 4 & 5 & 6 & 7 & 8 & 9 \\
\hline $\boldsymbol{k}$ & 2 & 6 & 8 & 5 & 6 & 4 & 2 & 8 & 3 \\
\hline $\begin{array}{c}\boldsymbol{n}^{\mathrm{CO}}{ }_{\max } / n^{\mathrm{E}} \\
\text { before flipping (\%) }\end{array}$ & 94.7 & 96.2 & 96.2 & 95.0 & 95.3 & 95.6 & 94.7 & 95.3 & 95.5 \\
\hline $\begin{array}{c}\boldsymbol{n}^{\mathrm{CO}}{ }_{\text {max }} / \boldsymbol{n}^{\mathrm{E}} \\
\text { after flipping (\%) }\end{array}$ & 96.7 & 97.9 & 97.4 & 97.4 & 97.6 & 97.7 & 96.7 & 97.1 & 97.6 \\
\hline
\end{tabular}

\section{Application to Larger Problems and Benchmark}

To demonstrate the performance of the algorithm, we applied it to larger problems. Tables 3 and 4 summarizes the value of $k$ required to maximize $n^{\mathrm{CO}}$ and $n^{\mathrm{CO}}$ max $/ n^{\mathrm{E}}$ before and after flipping for nine instances of problems with $\mathrm{N}=30$ and 40 , respectively. It is 
confirmed that lower eigenmodes provide good candidates and that the flipping process effectively improves the candidates. For $\mathrm{N}=30, n^{\mathrm{CO}}$ is a maximum at $k \leq 9$ and $n{ }^{\mathrm{CO}}$ max $/ n^{\mathrm{E}}$ after flipping is larger than $96.6 \%$. For $\mathrm{N}=40, n^{\mathrm{CO}}$ is a maximum at $k \leq 13$ and $n^{\mathrm{CO}}{ }_{\max } / n^{\mathrm{E}}$ after flipping is larger than $96.7 \%$. Overall, for $\mathrm{N}=10$ to 40 , the best solution can be found by checking the lowest $\sim \mathrm{N} / 2$ candidates.

Table 3. Values of $k$ required to maximize $n^{\mathrm{CO}}$ and $n^{\mathrm{CO}}$ max $/ n^{\mathrm{E}}$ before and after flipping for nine instances of problem with $\mathrm{N}=30$.

\begin{tabular}{cccccccccc}
\hline Sample\# & 1 & 2 & 3 & 4 & 5 & 6 & 7 & 8 & 5 \\
\hline$k$ & 7 & 2 & 2 & 7 & 9 & 2 & 5 & 2 \\
\hline $\begin{array}{c}n^{\mathrm{CO}} \\
\text { max }\end{array} n^{\mathrm{E}}$ & 95.9 & 94.9 & 95.3 & 95.4 & 95.8 & 95.1 & 95.7 & 95.4 & 95.2 \\
\hline $\begin{array}{c}n^{\mathrm{CO}} \\
\text { after flipping (\%) }\end{array}$ & 97.2 & 96.6 & 97.4 & 97.6 & 97.1 & 97.1 & 97.5 & 97.3 & 96.9 \\
\hline
\end{tabular}

Table 4. Values of $k$ required to maximize $n^{\mathrm{CO}}$ and $n^{\mathrm{CO}}$ max $/ n^{\mathrm{E}}$ before and after flipping for nine instances of problem with $\mathrm{N}=40$.

\begin{tabular}{ccccccccccc}
\hline Sample\# & 1 & 2 & 3 & 4 & 5 & 6 & 7 & 8 & 9 \\
\hline $\boldsymbol{k}$ & 7 & 1 & 13 & 6 & 1 & 10 & 10 & 4 & 94.9 & 94.9 \\
\hline $\begin{array}{c}n^{\mathrm{CO}} \text { max } / n^{\mathrm{E}} \\
\text { before flipping (\%) }\end{array}$ & 95.0 & 94.9 & 95.4 & 94.8 & 95.0 & 95.6 & 94.8 & 97.3 & 96.7 & 97.1 \\
\hline $\begin{array}{c}n^{\mathrm{CO}}{ }_{\max } / n^{\mathrm{E}} \\
\text { after flipping (\%) }\end{array}$ & 97.1 & 97.3 & 97.3 & 97.6 & 97.2 & 97.5 & 97.3 \\
\hline
\end{tabular}

As a benchmark study, the computation time is compared between the COS and a standard SA algorithm for ten instances of problems with $\mathrm{N}=50$. All trials were performed on a MacBook Pro with a 2.6-GHz Intel ${ }^{\circledR}$ Core i7 processor (6 cores) and 16 GB of RAM. The COS generated $n^{\mathrm{CO}}$ max in $2.74 \mathrm{~s}$, including the time required for the flipping process. Figure 4 shows the evolution of the number of satisfied couplings with iteration number in SA for a given problem. $n^{\mathrm{CO}}$ max and $n^{\mathrm{E}}$ are also indicated. After 27 iterations, SA provides a better solution than that provided by the COS. The computation time required to reach $n^{\mathrm{CO}}$ max (27 iterations) was $8.63 \mathrm{~s}$. Table 5 compares the computation time required to reach $n^{\mathrm{CO}}{ }_{\max }$ between the COS and SA for ten instances. Assuming that an $n^{\mathrm{CO}}$ max $/ n^{\mathrm{E}}$ value of $97 \%$ is satisfactory, the COS is three times faster than SA in generating the solution.

Table 5. $n^{\mathrm{CO}}{ }_{\max } / n^{\mathrm{E}}$ and computation time required to reach $n^{\mathrm{CO}}$ max with the $\mathrm{COS}\left(T_{\mathrm{CO}}\right)$ and SA $\left(T_{\mathrm{SA}}\right)$.

\begin{tabular}{ccccccccccccc}
\hline Sample\# & 1 & 2 & 3 & 4 & 5 & 6 & 7 & 8 & 9 & 10 \\
\hline $\boldsymbol{k}$ & 15 & 12 & 13 & 4 & 25 & 19 & 2 & 5 & 21 & 7 \\
\hline${ }_{n}{ }^{\mathrm{CO}}{ }_{\max } \boldsymbol{n}^{\mathrm{E}} \mathbf{( \% )}$ & 97.2 & 97.5 & 97.0 & 97.0 & 96.8 & 97.5 & 96.9 & 97.0 & 97.4 & 97.3 \\
\hline TCO & 2.74 & 2.71 & 2.74 & 2.73 & 2.75 & 2.74 & 2.73 & 2.73 & 2.76 & 2.74 \\
\hline TSA & 8.89 & 9.50 & 7.60 & 8.20 & 8.21 & 8.40 & 7.37 & 7.65 & 8.83 & 8.60 \\
\hline
\end{tabular}




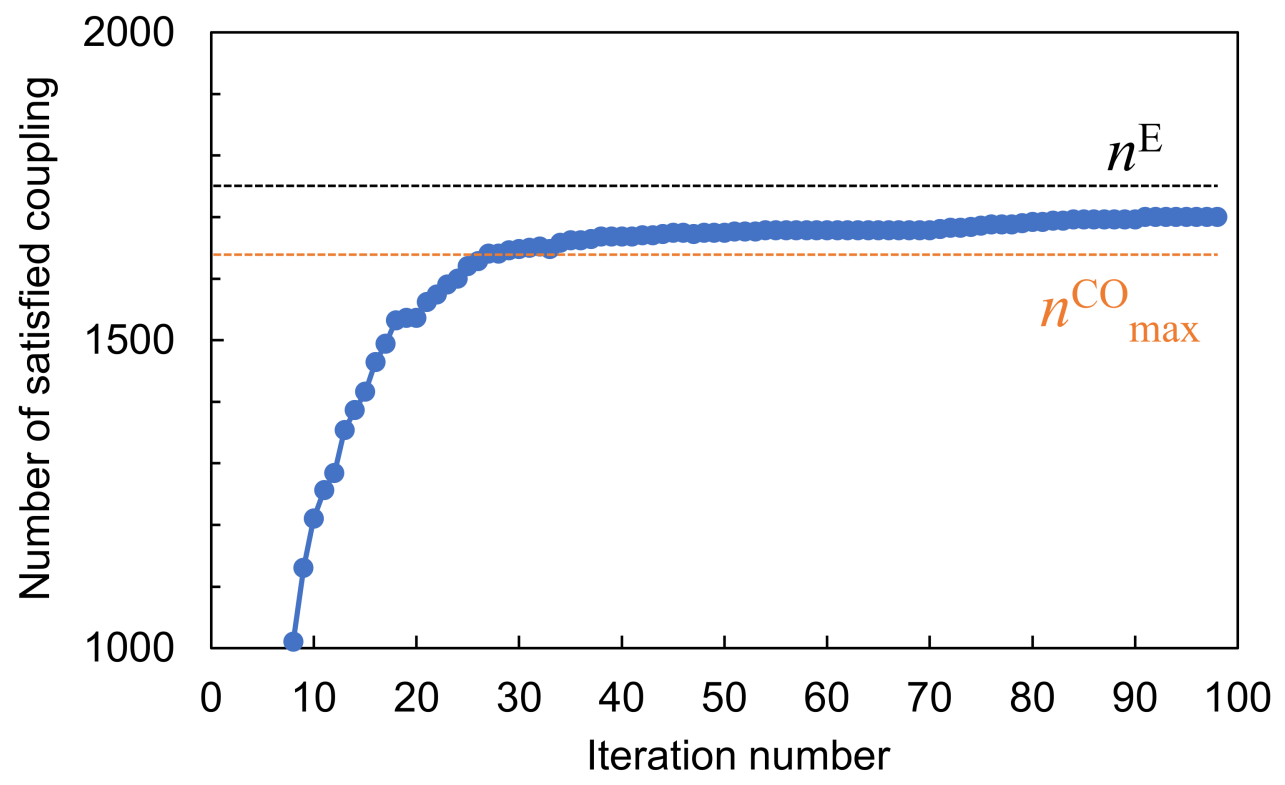

Figure 4. Evolution of the number of satisfied couplings with iteration number in SA for a given problem.

\section{Conclusions}

We evaluated the COS for Ising spin problems based on eigenmode characterization. After a square lattice $(\mathrm{N} \times \mathrm{N})$ Ising problem was converted to a coupled oscillator model that includes positive and negative coupling, the equation of motion, which was reduced to an eigenmode problem, was solved. For smaller problems $(\mathrm{N}=10)$, the oscillation amplitude (eigenvector) was delocalized (i.e., it covered the entire oscillator lattice) and a fairly good solution was obtained. For larger problems, the oscillation became localized, forming oscillator clusters with low frustration density (unfrustrated clusters). From a map of unfrustrated clusters for lower eigenmodes, we found that frustration tends to localize at the boundary between clusters. Frustration localization, where three of the four couplings are frustrated, is useful for reducing frustration by flipping the sign of the amplitude. Localization and the flipping method were applied to problems with $\mathrm{N}=40$. Good solutions with an accuracy of $97.2 \%$ in average (with respect to the exact ground state) were obtained simply by checking the lowest $13(\leq \mathrm{N} / 2)$ candidate eigenmodes. A benchmark study demonstrated that the computation time required to reach a fairly good solution $\left(n^{\mathrm{CO}}\right.$ max $)$ for the $\mathrm{COS}$ is three times shorter than that for $\mathrm{SA}$.

Author Contributions: Conceptualization, T.S.; software, S.M., O.I. and Y.H.; formal analysis, S.M., O.I. and Y.H.; investigation, S.M., O.I. and Y.H.; writing-original draft preparation, S.M.; writingreview and editing, T.S.; funding acquisition, T.S. All authors have read and agreed to the published version of the manuscript.

Funding: This work was partially supported by JSPS KAKENHI (grant number 18H04490 and 20H04645) and partially by MEXT Quantum Leap Flagship Program Grant No. JPMXS0118067246.

Institutional Review Board Statement: Not applicable.

Informed Consent Statement: Not applicable.

Data Availability Statement: The data presented in this study are available on request from the corresponding author.

Conflicts of Interest: The authors declare no conflict of interest.

\section{References}

1. Nishimori, H. Statistical Physics of Spin Glasses and Information Processing: An Introduction; Clarendon Press: Oxford, UK, 2001.

2. Mezard, M.; Parisi, G.; Virasoro, M. Spin Glass Theory and Beyond; World Scientific: Singapore, 1998. 
3. Ising, E. Beitrag zur Theorie des Ferromagnetismus. Z. Physik. 1925, 31, 253-258. [CrossRef]

4. Barahona, F. On the computational complexity of Ising spin glass models. J. Phys. A Math. Gen. 1982, 15, 3241-3253. [CrossRef]

5. Peres, F.; Castelli, M. Combinatorial Optimization Problems and Metaheuristics: Review, Challenges, Design, and Development. Appl. Sci. 2021, 11, 6449. [CrossRef]

6. Tanahashi, K.; Takayanagi, S.; Motohashi, T.; Tanaka, S. Application of Ising Machines and a Software Development for Ising Machines. Jpn. J. Appl. Phys. 2019, 88, 061010. [CrossRef]

7. Andrew, L. Ising formulations of many NP problems. Front. Phys. 2014, 2, 5.

8. Simone, C.D.; Diehl, M.; Jünger, M.; Mutzel, P.; Reinelt, G.; Rinaldi, G. Exact ground states of Ising spin glasses: New experimental results with a branch-and-cut algorithm. J. Stat. Phys. 1995, 80, 487-496. [CrossRef]

9. Blum, C.; Roli, A. Metaheuristics in combinatorial optimization: Overview and conceptual comparison. ACM Comput. Surv. 2003, 35, 268-308. [CrossRef]

10. Gendreau, M.; Potvin, J.Y. Metaheuristics in Combinatorial Optimization. Ann. Oper. Res. 2005, 140, 189-213. [CrossRef]

11. Kirkpatrick, S.; Gelatt, C.D.; Vecchi, M.P. Optimization by Simulated Annealing. Science 1983, 220, 671-680. [CrossRef] [PubMed]

12. Marinari, E.; Parisi, G. Simulated Tempering: A New Monte Carlo Scheme. EPL Europhys. Lett. 1992, 19, 451-458. [CrossRef]

13. Isakov, S.V.; Zintchenko, I.N.; Rønnow, T.F.; Troyer, M. Optimised simulated annealing for Ising spin glasses. Comput. Phys. Commun. 2015, 192, 265-271. [CrossRef]

14. Marandi, A.; Wang, Z.; Takata, K.; Byer, R.L.; Yamamoto, Y. Network of time-multiplexedoptical parametric oscillators as a coherent Ising machine. Nat. Photonics 2014, 8, 937-942. [CrossRef]

15. Inagaki, T.; Haribara, Y.; Igarashi, K.; Sonobe, T.; Tamate, S.; Honjo, T.; Marandi, A.; McMahon, P.L.; Umeki, T.; Enbutsu, K.; et al. A coherent Ising machine for 2000-node optimization problems. Science 2016, 354, 603-606. [CrossRef]

16. McMahon, P.L.; Marandi, A.; Haribara, Y.; Hamerly, R.; Langrock, C.; Tamate, S.; Inagaki, T.; Takesue, H.; Utsunomiya, S.; Aihara, K.; et al. A fully programmable 100-spin coherent Ising machine with all-to-all connections. Science 2016, 354, 614-617. [CrossRef] [PubMed]

17. Pierangeli, D.; Marcucci, G.; Conti, C. Large-scale photonic Ising machine by spatial light modulation. Phys. Rev. Lett. 2019, 122, 213902. [CrossRef]

18. Pierangeli, D.; Marcucci, G.; Brunner, D.; Conti, C. Noise-enhanced spatial-photonic Ising machine. Nanophotonics 2020, 9 , 4109-4116. [CrossRef]

19. Yamaoka, M.; Yoshimura, C.; Hayashi, M.; Okuyama, T.; Aoki, H.; Mizuno, H. A 20k-spin Ising chip to solve combinatorial optimization problems with CMOS annealing. IEEE J. Solid State Circ. 2015, 51, 303-309.

20. Tsukamoto, S.; Takatsu, M.; Matsubara, S.; Tamura, H. An accelerator architecture for combinatorial optimization problems. Fujitsu Sci. Tech. J. 2017, 53, 8-13.

21. Aramon, M.; Rosenberg, G.; Valiante, E.; Miyazawa, T.; Tamura, H.; Katzgraber, H.G. Physics-inspired optimization for quadratic unconstrained problems using a digital annealer. Front. Phys. 2019, 7, 48. [CrossRef]

22. Johnson, M.W.; Amin, M.H.S.; Gildert, S.; Lanting, T.; Hamze, F.; Dickson, N.; Harris, R.; Berkley, A.J.; Johansson, J.; Bunyk, P.; et al. Quantum annealing with manufactured spins. Nature 2011, 473, 194-198. [CrossRef]

23. Boixo, S.; Rønnow, T.F.; Isakov, S.V.; Wang, Z.; Wecker, D.; Lidar, D.A.; Martinis, J.M.; Troyer, M. Evidence for quantum annealing with more than one hundred qubits. Nat. Phys. 2014, 10, 218-224. [CrossRef]

24. Goto, H. Bifurcation-based adiabatic quantum computation with a nonlinear oscillator network. Sci. Rep. 2016, 6, 21686. [CrossRef] [PubMed]

25. Goto, H.; Tatsumura, K.; Dixon, A.R. Combinatorial optimization by simulating adiabatic bifurcations in nonlinear Hamiltonian systems. Sci. Adv. 2019, 5, eaav2372. [CrossRef] [PubMed]

26. Kanazawa, S.; Kuwamura, K.; Kihara, Y.; Hirukawa, Y.; Saiki, T. Computations with near-field coupled plasmon particles interacting with phase-change materials. Appl. Phys. A 2015, 121, 1323-1327. [CrossRef]

27. Saiki, T. Switching of localized surface plasmon resonance of gold nanoparticles using phase-change materials and implementation of computing functionality. Appl. Phys. A 2017, 123, 577. [CrossRef]

28. The Program is Available on the Server. Available online: https://software.cs.uni-koeln.de/spinglass/client.html (accessed on 6 September 2021).

29. Pardella, G.; Liers, F. Exact ground states of large two-dimensional planar Ising spin glasses. Phys. Rev. E 2008, $78,056705$. [CrossRef] 